\title{
EL DILEMA PARA DENUNCIAR: UNA CRÍTICA A \\ LA TRANSFERENCIA DE FUNCIONES DEL INDECOPI A SUSALUD EN MATERIA DE SERVICIOS MÉDICOS Y DE SEGUROS \\ THE DILEMMA TO COMPLAINT: A CRITIQUE OF THE \\ TRANSFER OF FUNCTIONS FROM INDECOPI TO SUSALUD IN \\ RESPECT OF MEDICAL AND INSURANCE SERVICES
}

\author{
Enzo Segovia Trocones* \\ Pontificia Universidad Católica del Perú
}

In this article, the author analyzes the distribution of functions between SuSalud and Indecopi, regarding the mandates of Legislative Decree 1158. In particular, the author addresses the functions of administrative regulation related to infractions in the provision of health services by the private sector in our national scope.

This is done taking into account considerations that are inherent to a fundamental right as the right to health, which implies an active duty for the State as a whole. This is the reason why intense criticisms are raised about the way in which the distribution of functions has been regulated among public administration bodies, through confusing rules that significantly increase the costs faced by consumers to present their complaints.

KEY WORDS: regulation; health; public administration; infringement; claim.
En el presente artículo, el autor analiza la distribución de competencias entre SuSalud y el Indecopi, a raíz de lo dispuesto por el Decreto Legislativo 1158. En particular, el autor aborda las funciones de regulación administrativa referidas a las infracciones en la prestación de servicios de salud por parte de privados en el ámbito nacional.

Ello se realiza a la luz de las consideraciones propias de un derecho fundamental que es el derecho a la salud, el cual implica un deber activo para el Estado en su conjunto. Bajo esta idea, se plantean duras críticas a la forma en que se ha normado la distribución de competencias entre los organismos de la administración pública, a través de normas poco claras que aumentan significativamente los costos que afrontan los usuarios para formular sus denuncias.

PALABRAS CLAVE: regulación; salud; administración pública; infracción; reclamo.

\footnotetext{
Alumno del último ciclo de pregrado en la Facultad de Derecho de la Pontificia Universidad Católica del Perú. El presente artículo fue elaborado para publicarse en una revista académica como parte del curso de Metodología de la Investigación Jurídica, con la asesoría del profesor Alejandro Moscol Salinas.
} de 2018, y aceptado por el mismo el 16 de junio de 2018. 


\section{INTRODUCCIÓN}

La salud no solo es un derecho amparado en nuestra Constitución, sino también se constituye en un presupuesto básico para el desarrollo de las demás actividades que realiza el ser humano, razón por la cual recibe una especial tutela de parte del Estado a fin de garantizar su acceso a todos e impedir que otros particulares o las mismas entidades estatales vulneren su pleno ejercicio.

De esta manera, este derecho se ha convertido en un deber para el Estado que se ha traducido en la creación de un Ministerio de Salud que se encarga de organizar y gestionar el Sistema Nacional de Salud, el cual integra organismos de otros ministerios como Essalud, las Fuerzas Armadas, la Policía Nacional y también entidades privadas como clínicas o centros médicos especializados, con la única finalidad de brindar un servicio de salud de buena calidad y accesible para todos.

En este sentido, el usuario de una clínica no es un consumidor cualquiera, pese a encontrarse en un mercado que funciona bajo las mismas condiciones que cualquier otro desde una perspectiva puramente económica, sino que se trata de un paciente que busca restablecer urgentemente su salud o la de sus parientes.

Es por ello que, con la finalidad de asegurar que los servicios de salud prestados en el país cumplan con un estándar de calidad y no pongan en riesgo la integridad o la vida de las personas, se creó la Superintendencia Nacional de Aseguramiento en Salud, la cual, a partir del 7 de diciembre de 2013, pasó a llamarse simplemente Superintendencia Nacional de Salud (SuSalud).

Hasta antes de esta fecha, los usuarios de servicios de salud solo podían acudir al Instituto Nacional de Defensa de la Competencia y de la Protección de la Propiedad Intelectual (Indecopi) en vía de protección al consumidor para reclamar por sus derechos, lo que limitaba las posibilidades de obtener una tutela adecuada, pues este organismo, además de conocer sobre regulación de la publicidad, eliminación de barreras burocráticas, registro de patentes, protección de derechos de autor, detección de cárteles y proteger el crédito mediante el sistema concursal, debía especializarse también en resolver controversias surgidas por la falta de idoneidad en los servicios médicos o productos de seguros.

Es por ello que el cambio de denominación traía también una transformación orgánica y funcional de SuSalud, a fin de especializarse no solo como un ente resolutivo de esta clase de controversias, sino también para reforzar sus funciones de super- visión y fiscalización de las entidades que se encontraban bajo su ámbito de competencia.

Sin embargo, los problemas de este cambio no se hicieron esperar y pronto los pacientes indignados por una mala atención médica o por una falta de reconocimiento de cobertura de su seguro se encontraron en medio de una disputa entre dos entidades del Estado que se supone debían velar por sus derechos. Por un lado, el Indecopi alegaba ya no ser competente para conocer dichas denuncias, mientras, por el otro, SuSalud rechazaba los reclamos sosteniendo que no se encontraba dentro de sus funciones atender dicha materia. En medio de todo ello, los pacientes y asegurados debían esperar a que las autoridades se pongan de acuerdo para hacer valer sus derechos quedando en completo estado de indefensión por tiempo indeterminado.

La importancia de este tema radica en que, hasta la fecha, sigue existiendo un desconocimiento generalizado de parte de los consumidores acerca de las funciones y competencias específicas de SuSalud, lo que conlleva a que no solo se haga un uso ineficiente de los recursos del Estado para atender los reclamos y denuncias declaradas improcedentes, sino también que los mismos administrados se vean perjudicados al encontrar justicia para sus casos luego de varios años o, en el peor de los casos, simplemente no encontrarla.

En este sentido, el propósito del presente trabajo es abordar el dilema que atraviesan los consumidores de servicios médicos y de seguros, desde el punto de vista del derecho administrativo y de la protección al consumidor e intentar esbozar una clasificación de productos y servicios sobre los que SuSalud o el Indecopi tienen competencia a fin de salvar la problemática interpretativa que surge de las normas pertinentes.

\section{PLANTEAMIENTO DEL PROBLEMA}

El 14 de agosto de 2015 entró en vigencia el Reglamento del procedimiento de transferencia de funciones del Indecopi a SuSalud en el marco de lo dispuesto en el Decreto Legislativo 1158 -Decreto Legislativo que dispone las medidas destinadas al fortalecimiento y cambio de denominación de la Superintendencia Nacional de Aseguramiento en Salud-, el cual pretendió regular la fase transitoria a fin de que el Indecopi dejara de ser competente para conocer denuncias derivadas de la vulneración de los derechos de los consumidores en materia de servicios de salud y seguros.

Sin embargo, esta transferencia de funciones no fue pacífica ni ordenada, pues en la práctica se co- 
menzaron a observar ciertos problemas de interpretación de la norma que están dejando actualmente en la deriva tanto a consumidores (pacientes y asegurados) como a los propios funcionarios de ambas instituciones públicas.

El problema que se pretende abordar en este artículo es la falta de precisión y claridad de las normas implicadas en la transferencia de las funciones que propone el Decreto Legislativo 1158, lo cual ha ocasionado una interpretación muy abierta y que ha devenido en conflictos de competencia administrativa.

De modo no exhaustivo, las siguientes son algunas normas que presentan problemas de interpretación al momento de ser aplicadas a casos concretos:

\section{Del Decreto Legislativo 1158:}

\section{Artículo 5: Ámbito de Competencia}

Artículo 6: De las Instituciones Administradoras de los Fondos de Aseguramiento en Salud.

- $\quad$ Artículo 7: De las Instituciones Prestadoras de Servicios de Salud

Inciso 16 del artículo 8: Funciones Generales

- $\quad$ Artículo 9: Funciones de la SuSalud referidas a las empresas de seguros y Afocat

Del Reglamento de Infracciones y Sanciones de SuSalud:

Todos los anexos de este reglamento en los que se tipifican las conductas infractoras susceptibles de sanción por SuSalud adolecen de una excesiva especificidad al momento de su tipificación.

Del Reglamento del Procedimiento de Transferencia de Funciones del Indecopi a SuSalud:

Artículo 5: Sobre las competencias generales de SuSalud

- Artículo 6: De las competencias sobre las IPRESS

- $\quad$ Artículo 7: De las competencias sobre SCTR

- $\quad$ Artículo 8: De las competencias sobre SOAT y Afocat

Artículo 9: De los procedimientos asumidos por SuSalud

- $\quad$ Artículo 10: De la aplicación del Código de Protección y Defensa del Consumidor
El conflicto de competencias de las dos entidades administrativas redunda principalmente en la disminución de la efectividad de la tutela que el consumidor debe recibir en situaciones de vulneración. Para ilustrar mejor esta situación, imaginemos el siguiente caso hipotético:

Usted es un trabajador que, al no encontrarse satisfecho con la atención que recibe del seguro social EsSalud, decide adquirir un seguro de salud privado. Meses después, sufre una fractura ósea a causa de un accidente que tuvo en su domicilio, por lo que llama a la ambulancia de la clínica afiliada a su seguro a fin de que le brinden la atención urgente que requiere. Es aquí donde comienzan los problemas, pues resulta que la ambulancia tardó más de una hora en llegar, pese a que la clínica no se encontraba a más de 10 cuadras de su domicilio; al llegar al servicio de emergencia de la clínica le indican que no es posible atenderlo, ya que su seguro de salud se encontraba anulado por la compañía aseguradora, por lo que tras intentar solicitar atención, los médicos de la clínica se negaron a atenderlo pues no consideraron una emergencia tal situación, indicándole que debía dirigirse por consulta externa ${ }^{1}$.

Al denunciar las infracciones, tendrá que tomar en cuenta que existen dos entidades administrativas potencialmente competentes para conocer su caso. Es así que decide interponer la denuncia ante el Indecopi; sin embargo, la Comisión de Protección al Consumidor 1 le indica que no es competente para conocer la negativa de atención en el servicio de emergencia ni la tardanza de la ambulancia en llegar al domicilio, admitiendo a trámite únicamente el extremo referido a la anulación del seguro por falta de pago, por lo que se ordena remitir una copia de todo lo actuado a SuSalud a fin de que esta entidad administrativa tome las medidas de su competencia.

Sin embargo, luego de analizar la denuncia, SuSalud resuelve admitir a trámite los extremos referidos a la negativa de atención en el servicio de emergencia y la indebida anulación de la póliza por supuesta falta de pago, declinando su competencia a la Comisión de Protección al Consumidor 2 en el extremo referido a la demora en la atención de

Tómese en cuenta para el análisis de este caso hipotético que las infracciones acaecieron luego de la entrada en vigencia del Decreto Supremo 026-2015-SA que aprueba el Reglamento de Transferencia de Funciones del Indecopi a Susalud, en el marco del Decreto Legislativo 1158. 
la ambulancia, toda vez que dicho servicio fue brindado por una empresa de transporte especializada, que no es considerada una IPRESS según la ley.

De este modo, su problema, el cual se originó de un solo hecho, ahora se encuentra divido en dos entidades administrativas, y en una de ellas tiene que seguir dos procedimientos paralelos, ya que estos están siendo tramitados por dos órganos resolutivos distintos. Sin comprender el porqué de tanto enredo burocrático y, lo peor de todo, habiéndose triplicado los costos que debe asumir para obtener una solución a su único problema. Ahora tendrá que pagar a su abogado por tres procedimientos distintos, pagar tres tasas administrativas distintas, presentar los documentos que se le requieran a tres órganos distintos y correr el riesgo de que se emitan resoluciones contradictorias.

\section{A. Algunos casos reales para comprender el problema}

Han pasado ya casi dos años desde que entró en vigencia el Reglamento de Transferencia de Funciones del Indecopi a SuSalud, en el marco del Decreto Legislativo 1158 (en adelante, Reglamento de Transferencia), por lo que, a la fecha, en principio, las denuncias en materias de servicios médicos deben ser declaradas improcedentes por haber prescrito el plazo para que el Indecopi pueda conocerlas y sancionarlas y las que se encuentren aún dentro del plazo, deberían ser remitidas a SuSalud. Sin embargo, ello no ha ocurrido así, pues como veremos a continuación, las competencias de ambas entidades administrativas no han quedado claramente delimitadas y las denuncias vienen y van como si se tratara de una pelota en una partida de tenis.

\section{a) Hostal La Marina S.R.L. vs. Sanitas Perú S.A} EPS

Hostal La Marina suscribió un contrato de prestación de servicios de seguridad social en salud para sus empleados; sin embargo, el 28 de diciembre de 2016 recibió una carta de Sanitas a través del cual le informó sobre el incremento del $50 \%$ en el costo de la prima. Posteriormente, Hostal La Marina se comunicó con Sanitas manifestando su desacuerdo con el incremento efectuado. Pese a ello, y sin tomar en cuenta su reclamo, la denunciada le remitió una factura por los servicios contratados con el incremento anunciado.
Ante esto, el denunciante interpuso un reclamo solicitando la anulación de su contrato. No obstante, dicha carta no fue respondida, por lo que acudió a SuSalud en búsqueda de una solución; sin embargo, le indicaron que dicha entidad no era competente para atender su reclamo, ya que el procedimiento llevado por la Intendencia de Derechos en Salud - IPROT estaba dirigida para personas que se enmarcan en la categoría de usuarios, entendiendo a estos como personas naturales, y terceros legitimados como asociaciones civiles con fines en salud. Sustentó dicha decisión en el artículo 1 del Reglamento para la Atención de Reclamos y Quejas de los Usuarios de IAFAS, IPRESS y UGIPRESS ${ }^{2}$ el cual indica que los usuarios y terceros legitimados pueden presentar quejas y reclamos.

Tras recibir la denuncia, la Comisión de Protección al Consumidor 1 del Indecopi, mediante Resolución Final 1130-2017/CC1, resolvió declarar improcedente la denuncia en la medida que SuSalud resultaba ser competente en virtud a que el Reglamento de Infracciones y Sanciones de SuSalud contemplaba como una infracción leve el supuesto referido a que una IAFAS no cumpla con los criterios definidos para el cálculo o reajuste de la siniestralidad de los planes contratados con los afiliados, los empleadores o quienes los representen, según lo establece SuSalud. Asimismo, con respecto al argumento relacionado a que SuSalud no se encontraba facultado para iniciar un procedimiento sancionador incoado por una persona jurídica, la Comisión determinó que, según el mismo Reglamento citado, estas pueden denunciar siempre que lo hagan en defensa de los derechos de sus trabajadores asegurados.

Presuntas infracciones:

Sanitas habría incrementado indebidamente el costo de la prima del contrato de prestación de servicios de seguridad social en salud suscrito por Hostal La Marina a favor de sus trabajadores.

Sanitas no habría atendido los reclamos interpuestos por Hostal La Marina.

b) Sra. Rosly Torres vs. Clínica Internacional S.A.

El 26 y 28 de marzo de 2014 la señora Torres sometió a su menor hijo a dos exámenes de tamizaje neonatal en la Clínica; sin embargo, cuando acudió a recoger los resultados, el proveedor denunciado solo le entregó el de uno de ellos, por lo que el

2 Véase el Decreto Supremo 030-2016-SA del 27 de julio de 2016 que aprueba el Reglamento para la Atención de Reclamos y Quejas de los Usuarios de IAFAS, IPRESS y UGIPRESS. 
16 de julio de 2015 interpuso un reclamo ante la misma Clínica sin obtener respuesta.

Posteriormente, la señora Torres decide presentar un reclamo ante SuSalud. Adicionalmente, esta entidad administrativa decidió remitir a la Comisión de Competencia Desleal del Indecopi una copia del expediente debido a que los hechos materia de denuncia ocurrieron el 26 de marzo de 2014 y el 16 de julio de 2015, sin brindar mayores alcances de tal decisión. A su vez, esta última remitió la denuncia a la Comisión de Protección al Consumidor 1.

\section{Presuntas infracciones:}

- La Clínica no habría entregado los resultados del examen practicado al hijo de la señora Torres.

- La Clínica no habría atendido el reclamo interpuesto por la señora Torres el 16 de julio de 2015.

c) Sra. Lita Arbulú vs. Asociación Peruano Japonesa

La señora Arbulú denunció ante el Indecopi que, el 14 de febrero de 2016, su menor hija fue internada por una infección estomacal en la Clínica, por lo que se le requirió que firmara un pagaré incompleto y el pago de S/ 157,48 por concepto de excedentes y gastos médicos no cubiertos de la hospitalización al momento del alta.

Solicitó a su compañía aseguradora, a través de la Clínica, la cobertura por el internamiento de su menor hija; sin embargo, el proveedor denunciado no le informó sobre el estado de su solicitud, teniendo que acudir personalmente a realizar las averiguaciones. En ese momento, la Clínica le indicó que la compañía aseguradora no había cubierto el tratamiento brindado a su hija, por lo que debía pagar el costo total del servicio. Asimismo, se le indicó que su pagaré había sido extraviado.

La Comisión de Protección al Consumidor 1, mediante Resolución Final 03452017/CC1 declaró improcedente la denuncia por falta de competencia, en la medida que las conductas cuestionadas por la señora Arbulú se encontraban contempladas en el Anexo III-A del Reglamento de Infracciones y Sanciones de SuSalud, razón por la cual se determinó remitir una copia de todo lo actuado a dicha entidad administrativa.

Posteriormente, SuSalud devolvió al Indecopi la denuncia de la señora Arbulú, toda vez que consideraba que no era la instancia administrativa com- petente para conocer su caso, sustentando su decisión en que los hechos descritos no configuraban elementos que se derivaran de una insatisfacción del servicio de salud o afectación del acceso a los servicios de salud de una persona, sino que se trataba esencialmente de una gestión administrativa de la IPRESS que contenía un pretensión de carácter patrimonial.

Presuntas infracciones:

- La Clínica no habría mantenido informada a la señora Arbulú sobre el estado de su solicitud de cobertura del internamiento de su menor hija.

La Clínica habría extraviado el título valor firmado en blanco por la señora Arbulú.

- La Clínica habría realizado un cobro indebido a la señora Arbulú por el tratamiento recibido en la Clínica.

De esta pequeña muestra de casos que pasaron tanto por los escritorios del Indecopi y SuSalud, se puede observar que las presuntas infracciones denunciadas van desde asuntos propiamente relacionados con la actividad de las entidades supervisadas, como la atención médica o el otorgamiento de cobertura, hasta actividades meramente administrativas como el cobro del servicio o la gestión de documentos. Asimismo, cabe resaltar que los conflictos negativos de competencia se producen por interpretaciones contradictorias de las normas que rigen la competencia administrativa de cada entidad, por lo que, de cara a proponer una solución, debemos centrar nuestra atención en formular una interpretación sistemática y armoniosa con la naturaleza de cada institución, sus fines y funciones asignadas a fin de entender un poco mejor la lógica que buscaba el legislador al momento de transferir parte de las competencias del Indecopi a SuSalud.

\section{B. Indecopi y SuSalud}

Ambas entidades son dos organismos técnicos especializados pertenecientes al Poder Ejecutivo que se encuentran regulados actualmente por los Decretos Legislativos 1033 y 1158.

Por un lado, tenemos que Indecopi se encuentra adscrito a la Presidencia del Consejo de Ministros, mientras que SuSalud hace lo mismo respecto del Ministerio de Salud. Esta diferencia es importante para el análisis, toda vez que la dependencia orgánica de cada una de estas instituciones nos brinda una noción preliminar de los límites y alcances de sus funciones y competencias. 
Ambos se encuentran categorizados como organismos técnicos especializados según el Decreto Supremo 058-2011-PCM ${ }^{3}$; sin embargo, cuentan con una organización distinta, pues mientras que en el Indecopi se observa una amplia variedad de órganos resolutivos encabezados por el Tribunal de Defensa de la Competencia y de la Propiedad Intelectual, el cual se encuentra en relación de coordinación con su Consejo Directivo, SuSalud cuenta también con un Tribunal de la Superintendencia, pero se aprecia mayor relevancia en órganos dedicados a la promoción, supervisión y fiscalización de derechos en salud.

Por otro lado, debemos resaltar que, en materia de protección al consumidor, el Indecopi cuenta con tres órganos resolutivos, y una de fiscalización los cuales son los siguientes:

a) El Órgano Resolutivo de Procedimientos Sumarísimos de Protección al Consumidor: fue concebido como una alternativa rápida para atender las denuncias cuya cuantía del bien o servicio cuestionado no supere las tres (3) unidades impositivas tributarias (UIT). Este órgano se subdivide en tres áreas a quienes se encargan distintas materias específicas.

b) La Comisión de Protección al Consumidor: es el órgano encargado de conocer las denuncias como primera instancia en las que se cuestionen productos y servicios cuya cuantía supere las tres (3) UIT. Además, constituye la segunda instancia respecto del Órgano Resolutivo de Procedimientos Sumarísimos.

c) La Sala Especializada en Protección al Consumidor: resuelve en segunda instancia las denuncias interpuestas ante la Comisión y forma parte del Tribunal del Indecopi, por lo que es considerada como la última instancia administrativa y con alcance nacional.

d) Gerencia de Supervisión y Fiscalización: tiene como función principal brindar apoyo técnico y ejecutivo a los órganos resolutivos o a sus Secretarías Técnicas con la finalidad de ejercer las actividades de supervisión y fiscalización en las actividades económicas determinadas.

Finalmente, SuSalud cuenta con distintos órganos que podemos agrupar según la función que desempeñan: a) Órganos de promoción y protección: encabezados por la Superintendencia Adjunta de Promoción y Protección de Derechos en Salud, constituidas por dos intendencias las cuales tienen a su cargo establecer, implementar y conducir las actividades orientadas a proteger los derechos en salud de los usuarios de las IAFAS e IPRESS, así como llevar el desarrollo de las auditorias en salud para la investigación de vulneraciones de los derechos de los usuarios. Asimismo, tienen por misión la difusión de los derechos en salud de los usuarios frente a las IPRESS e IAFAS a través de campañas de promoción e información de derechos y deberes.

b) Órganos de Inspección: encabezados por la Superintendencia Adjunta de Supervisión, a través de la Intendencia de Supervisión de IAFAS e IPRESS están encargados de velar por los procesos asociados a la calidad, oportunidad, disponibilidad y aceptabilidad en la prestación de servicios de salud, así como vigilar los aspectos contables y financieros de las entidades que se encuentran bajo su ámbito de competencia.

c) Órganos de Regulación y Fiscalización: a través de la Intendencia de normas y autorizaciones y la Intendencia de Fiscalización y Sanción, se encuentran encargados de velar por la restitución de los derechos en salud de los usuarios que se han visto vulnerados, así como de la adecuación del marco normativo que regula a las IAFAS. Se encuentran dirigidas por la Superintendencia Adjunta de Regulación y Fiscalización.

d) Órganos de solución de controversias

Tribunal de SuSalud: es el órgano que se encuentra encargado de resolver en última instancia administrativa los procedimientos administrativos sancionadores provenientes de las Superintendencias de Regulación y Fiscalización.

Centro de Conciliación y Arbitraje: es el órgano resolutivo que cuenta con autonomía técnica y funcional y que tiene como objetivo solucionar las controversias surgidas de las relaciones de consumo de los usuarios con las IPRESS e IAFAS, a través de mecanismos alternativos de solución de conflictos como el arbitraje y la conciliación. 
Las estructuras orgánicas de ambas instituciones nos brindan una guía para entender las funciones que han sido asignadas $y$, de este modo, entender la competencia material sobre las denuncias administrativas que reciben. Así, por ejemplo, podemos observar que el Indecopi, en materia de protección al consumidor, ha sido diseñado principalmente para la solución de controversias entre proveedores y consumidores de cualquier producto o servicio ofertado en el mercado, contando con un órgano de fiscalización muy poco activo, ya que, el grueso de procedimientos sancionadores tramitados por esta entidad procede de denuncias presentadas por los mismos usuarios afectados. En cambio, SuSalud cuenta con una estructura dedicada más a la fiscalización de los proveedores de servicios en salud y productos de seguros.

Esto puede explicar de manera preliminar por qué SuSalud es más reacia a aceptar denuncias administrativas de los usuarios cuya presunta infracción no calce exactamente con las infracciones tipificadas en su Reglamento de Infracciones y Sanciones, la cual, dicho sea de paso, pareciera haber sido diseñado a la medida de un fiscalizador o supervisor para imponer sanciones de oficio. Por el contrario, SuSalud dedica principalmente su actividad a realizar inspecciones y fiscalizaciones, a través de sus diversos órganos, a las clínicas, centros de salud y compañías aseguradoras iniciando, de este modo, procedimientos sancionadores de oficio que son resueltos finalmente por su Tribunal. A continuación, analizaremos las funciones asignadas a cada una de estas dos entidades para interpretar mejor las competencias trasladadas de Indecopi a SuSalud.

\section{LA FUNCIÓN ADMINISTRATIVA}

La función por antonomasia del Poder Ejecutivo es aquella que ejerce el Estado como gestor del interés público, a través de la cual, las personas jurídicas estatales entran en relación con los particulares, como sujetos de derecho (Brewer Carías, 2005). Pese a que el poder del Estado más idóneo para detentarla es el Ejecutivo, cierto sector de la doctrina considera que, tanto el Legislativo como el Judicial también la ejercen, aunque en menor medida.

Así, se pueden distinguir hasta dos concepciones de la función administrativa. La primera de ellas se encuentra agrupada bajo un criterio subjetivo u orgánico y sostiene que dicha función es aquella que ejerce el Poder Ejecutivo y todas las entidades y sujetos que actúan en su esfera. Esta tendencia conceptual inspirada en las ideas de García de Enterría ha sido duramente criticada por un sector doctrinario que reconoce la entidad jurídica y lógica del criterio material para definir la función administrativa (Cassagne, 2010).

La segunda concepción, sostiene que la noción de función administrativa es aquella que se caracteriza por su carácter concreto, su inmediatez y continuidad, por lo que dicha actividad no se circunscribiría únicamente a las acciones del Poder Ejecutivo, sino que también se admite la posibilidad de que los demás poderes del Estado la ejerzan. Los autores que comulgan con esta concepción objetiva hacen referencia a un aspecto teleológico que debe perseguir dicha función, el cual se podría considerar como la atención de los intereses púbicos que asume en los propios fines o la satisfacción de las necesidades colectivas o del interés público (Cassagne, 2010).

\section{Funciones asignadas a Indecopi}

Según el Decreto Legislativo 1033, las funciones asignadas al Indecopi, son, entre otras, las siguientes:

- Vigilar la libre iniciativa privada y la libertad de empresa mediante el control posterior y eliminación de barreras burocráticas ilegales e irracionales

\section{Defender la libre y leal competencia}

- Corregir las distorsiones en el mercado producidas por dumping y subsidios

- Proteger los derechos de los consumidores asegurando la idoneidad de bienes y servicios y evitar la discriminación

Facilitar el comercio exterior

Proteger el crédito mediante el sistema concursal

- Administrar el sistema de otorgamiento y protección de derechos de propiedad intelectual.

\section{Funciones asignadas a SuSalud}

Según el Decreto Legislativo 1158, las funciones asignadas a SuSalud son, entre otras, la de promover, supervisa, proteger y defender los derechos de las personas al acceso a los servicios de salud; conocer con competencia primaria y alcance nacional, las presuntas infracciones a las disposiciones relativas a la protección de los derechos de los usuarios en su relación de consumo con las IPRESS y/o IAFAS, incluyendo aquellas previas y derivadas de dicha relación; promover los mecanismos de conciliación y arbitraje para la solución de los conflictos suscitados entre los diferentes actores del Sistema Nacional de Salud; e, identificar las cláusulas abusivas en los contratos o convenios que suscriben las IAFAS con los asegurados. 
Por otro lado, en relación a las empresas de seguros y Afocat, el artículo 9 del citado Decreto Legislativo menciona que las funciones de SuSalud en relación a estas entidades se circunscriben a lo siguiente:

- Los procesos asociados a la presentación de servicios de salud en las IPRESS que brinden los servicios a los asegurados.

- El cumplimiento de las condiciones que se deriven de los convenios o contratos suscritos con los asegurados.

- $\quad$ El cumplimiento y la regulación en los contratos y convenios suscritos con las IPRESS; así como la oportunidad de pago a sus proveedores y prestadores en las siguientes materias: auditoria médica, guías de diagnóstico y tratamiento, códigos y estándares de información y solución de controversias.

En este punto es importante señalar que el artículo 9 nos brinda un límite de la competencia de SuSalud al circunscribir las funciones que se le había otorgado de manera general en el artículo 8 sobre las empresas de seguros y Afocat. De esta manera, debemos entender que, Indecopi y la Superintendencia de Banca, Seguros y AFP (SBS) mantienen sus competencias sobre dichos sujetos en el resto de materias.

\section{LA COMPETENCIA ADMINISTRATIVA}

La naturaleza jurídica y organización de ambas instituciones nos brinda una idea más cercana respecto de su finalidad y la misión con la cuales fueron concebidas al momento de su creación, siendo que, como sostiene Verónica Rojas, “(...) el funcionamiento de toda organización estatal se guía por objetivos nacionales predeterminados y se conduce hacia ellos a través del cumplimiento de las competencias" (Rojas, 2004, p.124). Así pues, para determinar la competencia de cada entidad del estado es necesario comprender primero sus funciones y la naturaleza de su diseño institucional, ya que el legislador debe asignar las competencias a las entidades que se encuentren en mejor capacidad de desarrollarlas. En este sentido la autora citada señala lo siguiente:

[...] no se puede entender a la competencia, y con ella a las porciones de poder de cada entidad pública, si es que no va de la mano con la planificación nacional, pues toda la organización de la administración pública -al final de cuentas- está orientada al fin supremo del Estado que es el servicio al ciudadano, a cuyos efectos se requiere una actuación conjunta de todas las organizaciones públicas que lo integran, las que precisamente actúan a través de sus competencias (Rojas, 2004, p. 124).

En este sentido, es pertinente recordar el concepto de competencia en el ámbito administrativo, el cual es definido en sentido amplio como "el poder reconocido no solo a un funcionario de crear actos jurídicos, sino también el de sujetarse a lo que la ley le permite, de manera tal que igual competencia tendrá el profesor que enseña, el ingeniero que abre carreteras, que el Gobernador de Departamento" (Vélez, 1979, p. 64).

Asimismo, Miguel Marienhoff señala que para considerar válido un acto administrativo se debe verificar si este ha sido emitido dentro de un "círculo de atribuciones legales" el cual determina la capacidad de la autoridad administrativa. De esta manera, la competencia administrativa puede ser definida como un complejo de funciones atribuido a un órgano administrativo o como la medida de la potestad atribuida a cada órgano (Marienhoff, 1966).

En esta misma línea, Agustín Gordillo sostiene que "la competencia es el conjunto de funciones que un agente puede legítimamente ejercer" (GordiIlo, 2000, p. XII-7). Asimismo, destaca la distinción existente entre la competencia y el ejercicio de la función señalando que no debe confundirse la competencia con la "aptitud de obrar" de los órganos administrativos, o con el "complejo de atribuciones otorgadas al órgano", pues la competencia designa únicamente al conjunto de actividades que el órgano puede legítimamente realizar (Gordillo, 2000, pp. XII-11).

Ahora bien, la discusión del problema que nos encontramos abordando en el presente trabajo se centra en la competencia que le correspondería tanto a Indecopi como a SuSalud en razón del objeto material de las denuncias recibidas por cada entidad, es decir qué servicio o producto se está discutiendo o cuestionando por el consumidor. Al respecto, Juan Carlos Cassagne realiza una clasificación de la competencia administrativa en función al grado, lugar, tiempo y materia, sosteniendo particularmente sobre este último que se trata de "[...] la competencia por la cual el derecho objetivo (constitución, ley, reglamento) adjudica una serie de funciones y atribuciones a los órganos y sujetos estatales para la realización de las tareas que se le encomienden. Como ya hemos visto, rige en esta cuestión el principio de especialidad, por cuyo mérito cada órgano o sujeto estatal tiene competencia para realizar todo aquello que se encuentre vinculado a los fines de su creación" (Cassagne, 1981, pp. 191-192). 
En esta línea, el autor señala que la incompetencia por razón de materia importa la nulidad absoluta del acto administrativo y, como tal, no susceptible de saneamiento. El fundamento de ello no solo radica en el principio de división del trabajo que rige a la administración pública, sino también en la necesidad de adecuar la actividad administrativa a reglas jurídicas preestablecidas (Cassagne, 1981). De este modo, Cassagne sostiene que "en el plano externo y objetivo la competencia en razón de la materia importa entonces una delimitación de las atribuciones de los sujetos y órganos estatales establecidos en garantía del administrado y orientadas por el principio de la especialidad, cuya violación da lugar a la nulidad absoluta del acto" (1981, pp. 282-283).

En un sentido más específico, consideramos que la competencia en el ámbito administrativo se refiere al poder otorgado a los órganos y funcionarios de la administración para emitir actos jurídicos unilaterales sobre los particulares en determinados ámbitos fijados por la ley (Vélez, 1979), en específico para el caso bajo estudio, en razón de la materia de los reclamos o denuncias administrativas presentados ante el Indecopi o SuSalud.

Estas materias han sido fijadas por la ley y desarrolladas por el reglamento, y deben leerse a la luz de las funciones generales asignadas a cada institución estatal, pues, como señalamos, la división de las materias no solo significa una garantía al ciudadano al ofrecerle seguridad jurídica respecto de cuál será la entidad que administrará su caso, sino también tal división debe realizarse bajo el principio de especialidad, el cual toma en cuenta la capacidad de cada institución para llevar un procedimiento de la forma más idónea y eficiente. A continuación, delimitaremos, a groso modo, dichos ámbitos en los que ambas instituciones pueden ejercer sus funciones.

\section{1. Ámbito de competencia del Indecopi}

En materia de protección al consumidor, según el artículo 105 del Código de Consumo, Indecopi cuenta con competencia primaria y de alcance nacional para conocer las presuntas infracciones a las disposiciones de este cuerpo normativo, así como para imponer sanciones y medidas correctivas conforme al Decreto Legislativo 1033, ley de Organización y Funciones del Indecopi.

Es importante resaltar que "competencia primaria y de alcance nacional" debe entenderse como la función supletoria competencial que esta institución goza respecto de otras entidades administrativas. Es decir, en tanto la ley no haya asignado de manera expresa competencia a otro organismo público para proteger y defender los derechos de los consumidores en una determinada materia, el Indecopi será la entidad competente.

Ejemplos de esto son las competencias que se le han asignado a la Superintendencia de Banca Seguros y Administradoras Privadas de Fondos de Pensiones en materia de pensiones de AFP's, al Organismo Supervisor de la Inversión Privada en Telecomunicaciones en materia de servicios de telefonía fija, móvil, internet o televisión por cable, y SuSalud para servicios médicos y de seguros.

Este criterio ha sido recogido por el Tribunal del Indecopi en la resolución 02771999/TDC-INDECOPI el cual señala lo siguiente:

Por excepción establecida en norma expresa de rango legal, únicamente pueden entenderse aquellas disposiciones contenidas en las leyes, u otras normas de igual jerarquía, que señalen que una entidad administrativa, distinta a la Comisión de Protección al Consumidor del Indecopi, será competente para sancionar presuntas infracciones (a la Ley de Protección al Consumidor) que puedan cometerse en las relaciones de consumo que se presenten en un sector específico ${ }^{4}$.

\section{2. Ámbito de competencia de SuSalud}

En materia de protección de los derechos de los usuarios de servicios médicos y de seguros, el Decreto Legislativo 1158 establece que SuSalud tiene por objeto promover, defender y proteger los derechos de quienes accedan a los servicios de salud garantizando que dichos servicios sean de calidad.

Por otro lado, dicha norma establece un primer criterio subjetivo de competencia al señalar que las Instituciones Administradoras de Fondos de Aseguramiento en Salud (IAFAS), las Instituciones Prestadoras de Servicios de Salud (IPRESS) y las Unidades de Gestión de IPRESS se encuentran bajo su ámbito de competencia.

En este sentido, se debe entender por IAFAS a todas aquellas entidades que reciban, capten y/o gestionen fondos para la cobertura de las atenciones de salud o que oferten cobertura de riesgos de salud bajo cualquier modalidad. A modo de ejem-

4 Ver Resolución 277-1999/TDC-INDECOPI del 18 de agosto de 1999, seguido por Shirley Sánchez Cama contra José Cantuarias Pacheco y Corporación José R. Lindley S.A. 
plo, la norma señala una lista de entidades públicas y privadas entre las que se pueden destacar a las Entidades Prestadoras de Salud (Pacífico EPS, Rímac EPS, etc.), las empresas de seguros (Mapfre, La Positiva, Oncosalud, etc.) y las Asociaciones de Fondos Regionales y Provinciales Contra Accidentes de Tránsito (Afocat).

Por otro lado, siguiendo el criterio subjetivo, también se encuentran bajo el ámbito de competencia de SuSalud, las IPRESS que en términos generales agrupan a todos los hospitales, clínicas y demás entidades, sean estas públicas, privadas o mixtas que se caracterizan por realizar atenciones de salud con fines de prevención, promoción, diagnóstico, tratamiento y/o rehabilitación.

En este punto, el Decreto Legislativo hace una importante precisión respecto de los servicios brindados en IPRESS que, a prima facie, no parecieran ser materia de competencia de SuSalud. Y es que la norma refiere que esta institución no solo podrá conocer las controversias surgidas a raíz de la mala prestación de los servicios esenciales que brinda una IPRESS, sino también sobre aquellos que tienen por finalidad coadyuvar a la prevención, promoción, diagnóstico, tratamiento y/o rehabilitación de la salud los cuales son considerados como servicio auxiliares o complementarios. De conformidad con esta disposición, se debe entender que desde una falta de idoneidad en el servicio de transporte en ambulancia hasta el mal servicio brindado por el personal de seguridad de la clínica pueden ser cuestionados ante SuSalud, en la medida que pueden calificar como servicios que coadyuvan al servicio principal brindado por la IPRESS.

Así, podemos identificar un segundo criterio empleado en la norma para delimitar el ámbito de competencia de esta institución: el criterio material. No solo basta saber ante qué sujeto nos encontramos, sino también qué actividad realiza y con qué finalidad. En este sentido, es pertinente citar el artículo 7 y 8 del Reglamento del Procedimiento de Transferencia de Funciones del Indecopi a SuSalud, los cuales señalan que, respecto del Seguro Complementario de Trabajo de Riesgo, SuSalud será competente para supervisar el cumplimiento de las normas que protegen a los consumidores en materia referida a la cobertura y prestaciones de salud. Asimismo, SuSalud es competente, en principio, para supervisar y hacer cumplir las normas de protección al consumidor sobre el Seguro Obligatorio de Accidentes de Tránsito (SOAT) y Certificado contra Accidentes de Tránsito (CAT), ofertadas por las Afocat, salvo en lo referente a cobertura en casos de muerte, invalidez permanente, incapacidad temporal y gastos de sepelio, los cuales serán competencia del Indecopi y la SBS.

\section{HACIA UNA CATEGORIZACIÓN DE LOS SER- VICIOS Y SUS ENTIDADES COMPETENTES}

El núcleo del problema radica básicamente en que los hechos que generan una denuncia contra una clínica o una compañía aseguradora son situaciones complejas en las que concurren distintos actos de servicios brindados por diferentes proveedores (usualmente privados), quienes ofrecen sus servicios, los cuales han sido diseñados pensado no solo en que el paciente se cure de su enfermedad, sino también que se sienta seguro al llegar a las instalaciones de la clínica, que reciba buena alimentación mientras se encuentra hospitalizado, que la administración gestione adecuadamente las historias clínicas, que exista buena comunicación con la empresa de seguros, etc.

En esta línea, la normativa que determine las competencias de las entidades administrativas encargadas de velar por el cumplimiento y respeto de los consumidores y usuarios en esta materia debe ser clara a fin de evitar que se traslade el costo de tener que interpretarlas a los agentes económicos más débiles del mercado.

El siguiente cuadro representa el espectro de productos y servicios ofrecidos en el mercado que son susceptibles ser materias de competencia de SuSalud y que se encuentran ordenadas desde aquellos sobre que no generan mayor duda hasta los que deben ser analizados caso por caso. Ver gráfico 1.

\section{Actos médicos}

Los actos médicos encierran todas aquellas actividades realizadas por los profesionales de la salud que tienen como finalidad diagnosticar, tratar o prevenir una enfermedad. Constituyen la parte del espectro de servicios ofrecidos por las IPRESS en las que casi no existe discusión alguna respecto de la competencia, siendo que, tanto SuSalud como el Indecopi concuerdan en que la competencia de dicha materia le corresponde a la primera. De esta manera, si un paciente reclama un mal diagnóstico, una pésima intervención quirúrgica o un medicamento mal recetado que no sirvió para su tratamiento deberá dirigirse sin duda alguna a SuSalud para denunciar el hecho, claro está, siempre que la conducta infractora haya sucedido luego del 13 de agosto de 2015.

\section{Seguros y planes de salud}

En relación a los productos de seguros y planes de salud que ofertan diversos tipos de IAFAS como las empresas de seguros o las entidades prestadoras de salud, el Reglamento de Transferencia ha sido 


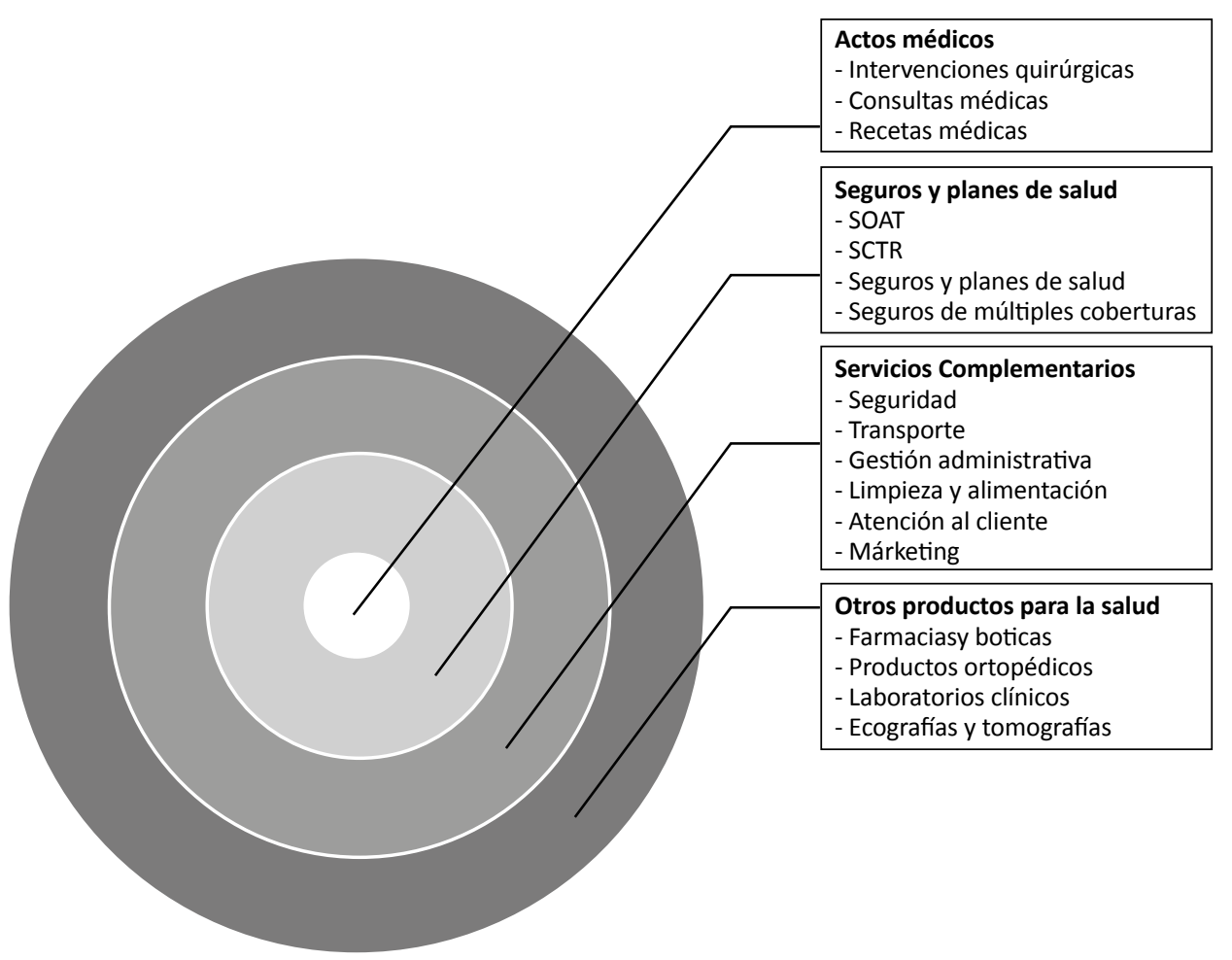

claro en establecer en los artículo 7 y 8 que SuSalud es competente para conocer todas las denuncias que versen sobre el Seguro Complementario de Trabajo de Riesgo y el Seguro Obligatorio de Accidentes de Tránsito salvo en lo referido a prestaciones económicas como las coberturas por gastos de sepelio o las pensiones de sobrevivencia, invalidez permanente o incapacidad temporal, materias sobre las cuales mantiene la competencia el Indecopi y la SBS. Sin embargo, la norma no aclara qué sucede con las coberturas de otros tipos de seguros que tengan por finalidad financiar un acto médico o entregar una suma alzada por el diagnostico de alguna enfermedad.

Para ello, es preciso remitirnos a lo indicado en el artículo 9 del Decreto Legislativo 1158 el cual señala que las funciones de SuSalud sobre las empresas de seguros ${ }^{5}$ y Afocat, se circunscriben a los siguientes supuestos:

a) Los procesos asociados a la prestación de servicios de salud en las IPRESS que brinden los servicios a los asegurados.

Este es el supuesto más básico que contempla la norma y está referida esencialmente a que el usuario podrá denunciar ante SuSalud cualquier conducta cuestionable que surja con motivo de la prestación de un servicio de salud en las clínicas afiliadas a la compañía aseguradora con la cual contrató una póliza de seguro. El típico caso de este supuesto lo podemos observar cuando un paciente no recibe la atención médica solicitada debido a que la compañía aseguradora no emitió la carta de garantía correspondiente.

b) El cumplimiento de las condiciones que se deriven de los convenios o contratos suscritos con los asegurados o con las entidades que las representen.

Desde una primera lectura, pareciera que cualquier incumplimiento que se derive del contrato de seguro suscrito con la aseguradora permitiría interponer un reclamo o una denuncia ante SuSalud; sin embargo, este supuesto se debe entender con la limitación de competencia ya establecida por el supuesto anterior; es decir, que dicha entidad administrativa solo será competente para conocer los casos que versen sobre incumplimientos de las condiciones del contrato

5 Cabe advertir que el citado Decreto Legislativo no precisa si las llamadas "empresas de seguros" referidas en este artículo son las contempladas en los incisos 6 del artículo 6 del mismo cuerpo normativo o si se refiere a las IAFAS en general. 
de seguro siempre que se encuentren relacionados con la prestación de un servicio de salud en una de sus IPRESS afiliadas. Lo que significa, en principio, que, si soy titular de un seguro que me brinda una suma alzada por el primer diagnóstico de cáncer, pero dicho monto no se encuentra destinado exclusivamente para el financiamiento de la intervención quirúrgica o el tratamiento que recibiría en el futuro, SuSalud no es competente para conocer dicho caso.

c) El cumplimiento y la regulación de los contratos o convenios suscritos con las IPRESS, así como la oportunidad de pago a sus proveedores y prestadores, será materia de regulación en los contratos los siguientes aspectos: auditoria médica, guías de diagnóstico y tratamiento, códigos y estándares de información y solución de controversias.

Mediante este último supuesto un usuario podría denunciar ante SuSalud desde una falta de atención a un reclamo, hasta la emisión de una carta de garantía que no cubre el tratamiento médico requerido por el paciente, siempre que estas conductas provengan de las prestaciones pactadas en los convenios suscritos entre la IPRESS y la empresa de seguros que incidan en la idoneidad del servicio brindado al consumidor.

\section{Servicios complementarios}

En relación a los servicios complementarios, se trata de uno de los supuestos más grises en los que no queda claro a qué entidad administrativa le corresponde la competencia para conocer los cuestionamientos de dichos servicios. Este es el caso de la señora Arbulú quien denunció a la Asociación Peruano Japonesa por no haberla mantenido informada sobre el estado de su solicitud de cobertura del internamiento de su menor hija, así como por haber extraviado el pagaré incompleto que había suscrito a solicitud de la clínica. SuSalud rechazó la admisión de este tipo de denuncias aduciendo que se trataban de cuestiones meramente administrativas y patrimoniales que no redundaban en una mala prestación del servicio médico o que implicara una insatisfacción del usuario.

Cabe recordar que los servicios complementarios son aquellos que una IPRESS o IAFAS brindan, ya sea de manera directa o contratando a un tercero, a fin de mejorar o coadyuvar a la prestación del servicio o producto principal para la cual fueron contratadas. Por ejemplo, una clínica brinda esencialmente prestaciones de salud como intervenciones quirúrgicas, hospitalizaciones, consultas especializadas, etc.; sin embargo, no es posible imaginar la prestación de estos servicios sin que dicha clínica cuente con un servicio de vigilancia o seguridad, limpieza, alimentación para los pacientes internados y, sobre todo, una buena gestión administrativa. En el caso de una compañía de seguros, si bien no es esencial para los productos que ofrece, un consumidor espera que estas empresas cuenten con disponibilidad de ambulancias, grúas mecánicas, una buena plataforma presencial y telefónica de atención al cliente, etc.

La falta de idoneidad en estos servicios complementarios implicaría necesariamente que el usuario o consumidor haya contratado el producto principal que lo determine en una relación de consumo con la clínica o la compañía aseguradora. Por tales motivos, el denunciante de estos hechos cuestionará todas las conductas en conjunto y no algunos por separado, por lo que resulta totalmente razonable que la entidad administrativa que reciba dicha denuncia atienda y resuelva la controversia de manera íntegra.

Así, el inciso 1 del artículo 8 del Decreto Legislativo 1158 señala que es una función de SuSalud la siguiente:

Promover, proteger y defender los derechos de las personas al acceso de los servicios, supervisando que las prestaciones sean otorgadas con calidad, oportunidad, disponibilidad y aceptabilidad, con independencia de quien las financie, así como los que correspondan en su relación de consumo con las IAFAS o IPRESS incluyendo aquellas previas y derivadas de dicha relación. (Subrayado añadido).

Asimismo, el inciso 16 del artículo 8 del mismo Decreto Legislativo establece como otra función a cargo de SuSalud lo siguiente:

Conocer con competencia primaria y alcance nacional, las presuntas infracciones a las disposiciones relativas a la protección de los derechos de los usuarios en su relación de consumo con las IPRESS y/o IAFAS, incluyendo aquellas previas y derivadas de dicha relación. (Subrayado añadido).

Por otro lado, el Reglamento de Transferencia también reguló lo establecido en el Decreto Legislativo 1158 reiterando, en su artículo 9, que SuSalud asume las competencias relativas a la protección de los derechos de los usuarios en su relación de consumo con las instituciones bajo su ámbito de competencia, así como aquellas previas y derivadas de estas. 
No cabe duda entonces que el legislador estuvo pensando en los servicios complementarios cuando redactó la frase "previas y derivadas de dicha relación", ya que como se ha explicado, un tipo de relación previa a la relación de consumo principal con una IPRESS sería por ejemplo el servicio de atención al cliente que recibiría una persona cuando acude a una clínica a solicitar información sobre el costo, forma y tiempo que le implicará una intervención quirúrgica que necesita con suma urgencia. En este sentido, si la persona que le atiende comete una falta de idoneidad, discriminándolo o brindándole información incorrecta, el usuario puede cuestionar el hecho ante SuSalud presentándola como una relación previa a la prestación del servicio principal.

Del mismo modo, si la relación de consumo ya se ha entablado al haber sido operado sin mayor inconveniente, pero, debido a un mal lavado de los alimentos que le proporcionaron en la clínica cuando estuvo hospitalizado para su recuperación, contrajo una enfermedad estomacal, también podrá cuestionar la idoneidad de dicha conducta denunciándolo como una relación derivada del servicio principal.

En el caso de la señora Arbulú, si bien el servicio cuestionado a la Asociación Peruano Japonesa no se encontraba referido a algún acto médico o servicio propio de la clínica, SuSalud debió admitir a trámite su denuncia en la medida que la falta de información sobre el estado de su solicitud de cobertura y el extravío del pagaré incompleto constituyen relaciones de consumo derivadas de la prestación principal consistente en el internamiento de su menor hija y tratamiento de su infección estomacal.

El Indecopi ya se ha pronunciado respecto de las relaciones que surgen de manera previa a entablarse una relación de consumo en la Resolución Final 674-2015/ILN-CPC, en la que un padre de familia denunció a una clínica por negarle la atención a su menor hijo, debido a que no contaba con un médico pediatra. En su defensa, la clínica señaló que el padre se negó a seguir el procedimiento de atención. La Comisión declaró fundada la denuncia, pues a través de una constatación policial se acreditó que, al momento del requerimiento de atención, el establecimiento no contaba con un especialista en pediatría ${ }^{6}$.

Asimismo, cabe precisar que en el concepto de relación previa de consumo se encuentran inclui- da la noción de consumidor expuesto el cual ha sido ampliamente tratado en la jurisprudencia del Tribunal del Indecopi, concluyéndose que dicho consumidor también es merecedor de tutela, pese a no haber adquirido un seguro o haber contratado los servicios de una IPRESS. Así, si una persona homosexual acude a la clínica a acompañar a su pareja internada y es expulsada debido a las "inapropiadas muestras de afecto" también podría denunciar este hecho, en la medida que se encuentra expuesto a una relación de consumo, pues, como sostiene Espinoza, "no debemos olvidar que el consumidor no deja de ser una persona que interactúa en el mercado y, en atención al artículo 3 de la Constitución, merece una amplia protección y tutela, que no debe ser limitada a la concreción de una relación de consumo" (Espinoza, 2011, p. 165).

Como último punto, es pertinente mencionar el caso del servicio de marketing del cual requieren tanto las IPRESS como las IAFAS para promocionar sus productos y servicios. Estos constituyen también casos de exposición a una relación de consumo, en la medida que los potenciales usuarios o consumidores se ven expuestos a actividad publicitaria o promocional como la de una clínica especializada en atención ginecológica y obstétrica que realiza llamadas telefónicas a los domicilios para ofrecer programas de salud para el cuidado de mujeres durante la etapa de gestación hasta el parto. Estas conductas también son susceptibles de ser cuestionadas; sin embargo, debemos recordar que las denuncias podrán ser interpuestas ante SuSalud o el Indecopi dependiendo de quién es proveedor denunciado, ya que, como se explicó líneas arriba, para el caso de las IAFAS, SuSalud solo es competente si la conducta cuestionada se enmarca en alguno de los tres supuestos señalados en el artículo 9 del Decreto Legislativo 1158.

\section{Otros productos y servicios relacionados con la salud humana}

Esta última parte del espectro de servicios y productos bajo análisis comprende a aquellas que, sin pertenecer necesariamente a la estructura organizacional de una IPRESS o IAFAS, se constituyen como establecimientos particulares que ofrecen dichos servicios de manera independiente y con determinadas restricciones.

Pertenecen a este grupo, la venta de productos farmacéuticos, ortopédicos, de medicina alternativa,

$6 \quad$ Cfr. Lineamientos de Protección al Consumidor 2016. 
servicios de análisis de laboratorio, ecografías, tomografías, resonancias magnéticas, venta de insumos químicos y equipos médicos u odontológicos, etc. Muchos de los establecimientos que brindan estos servicios también son pasibles de cometer conductas infractoras al deber de idoneidad o de información; sin embargo, no todas pueden ser cuestionadas ante SuSalud.

En este punto es pertinente revisar el artículo 7 del Decreto Legislativo 1158 el cual nos brinda la definición de IPRESS de la siguiente manera:

Las Instituciones Prestadoras de Servicios de Salud (IPRESS) son aquellos establecimientos de salud y servicios médicos de apoyo, públicos, privados o mixtos, creados o por crearse que realizan atención de salud con fines de prevención, promoción, diagnóstico, tratamiento y/o rehabilitación; así como aquellos servicios complementarios y auxiliares de la atención médica, que tienen por finalidad coadyuvar en la prevención, promoción, diagnóstico, tratamiento y/o rehabilitación de la salud.

En adición al cumplimiento de las normas de carácter general del Ministerio de Salud, para brindar servicios de salud deberán encontrarse registradas en la Superintendencia Nacional de Salud. (Subrayado añadido)

En el caso de las farmacias, boticas y centros de venta de insumos químicos y equipos médicos $u$ odontológicos (desde sillas para discapacitados, frenos dentales, hasta balones de oxígeno medicinal), si bien son actividades que coadyuvan en la prevención, tratamiento y rehabilitación de la salud, siempre que se trate únicamente de un acto de compraventa, no se encuentran bajo el ámbito de competencia de SuSalud, toda vez que no involucra el ejercicio de una atención médica, razón por la cual a estos establecimientos no pueden categorizárselas como IPRESS.

De esta manera, mediante Resolución 2280-2016/ CC1, la Comisión de Protección al Consumidor 1 declinó su competencia a la Comisión de Protección al Consumidor 2 en tanto consideró que no era competente para conocer la denuncia inter- puesta por el consumidor disconforme contra una empresa dedicada a la venta de balones de oxígeno medicinal por negarse injustificadamente a seguir proveyéndole de dicho producto. Asimismo, señaló que esta actividad comercial relacionada a la venta de productos farmacéuticos y médicos no se enmarcaba en ninguna de las competencias atribuidas por el artículo 1 de la Resolución 027-2013-INDECOPI/COD ${ }^{7}$.

Una mención especial merece el caso de las farmacias las cuales se encuentran impedidas de realizar consultas médicas, análisis clínicos, campañas médicas, albergar consultorios médicos, recolectar muestras para laboratorios clínicos, así como la venta de cualquier otro producto o la prestación de servicios que puedan contaminar el ambiente del establecimiento farmacéutico. Esta prohibición, establecida por la Resolución Directoral 006-2015-DIGEMID-DG-MINSA, refuerza el argumento de que las farmacias solo se encuentran autorizadas para el expendio de medicamentos, razón por la cual no pueden enmarcarse dentro de la categoría de IPRESS.

Para el caso de los establecimientos de venta de productos y servicios de medicina alternativa como hierbas, materiales y preparaciones herbarias, productos herbarios acabados, actividad terapéutica como la acupuntura o la quiropraxia, la venta de productos con principios activos, si bien pueden ser considerados como servicios médicos que tienen por finalidad el diagnóstico, tratamiento y/o rehabilitación de la salud, debemos recordar que según la Organización Mundial de la Salud, la medicina complementaria o alternativa es aquella que refieren a un "conjunto amplio de prácticas de atención de salud que no forman parte de la propia tradición del país y no están integradas en el sistema sanitario principal" (OMS, 2017). Por esta razón, si un usuario decide realizarse una sesión de acupuntura para aliviar sus dolores lumbares crónicos y este no da resultados, no podrá denunciar la idoneidad de dicho servicio ante SuSalud, ya que la definición de IPRESS no alcanza a este tipo de establecimientos al no ser considerados parte del Sistema Nacional de Salud de nuestro país ${ }^{8}$.

Finalmente, respecto a los establecimientos que brindan servicios de diagnóstico por imágenes y

\footnotetext{
Resolución del 10 de febrero de 2013 mediante el cual se aprueba la división temática de la competencia resolutiva de las Comisiones de Protección al Consumidor 1 y 2 de la Sede Central

8 Para mayor información sobre el Sistema Nacional de Salud del Perú véase: SÁNCHEZ-MORENO, Francisco: El Sistema Nacional de Salud en el Perú. En Revista Peruana de Medicina Experimental y Salud Pública. Lima. Consulta: 6 de julio de 2017. <http://www.scielo.org.pe/scielo.php?pid=S1726-46342014000400021\&script=sci_arttext>
} 
los laboratorios clínicos se encuentran comprendidos en la categoría de IPRESS, en la medida que brindan servicios médicos de apoyo realizando atenciones médicas con fines de prevención y diagnóstico de enfermedades. Estos establecimientos cuentan con profesionales de la salud especializados y capacitados para el manejo de los equipos médicos quienes no solo se limitan al uso de dichas herramientas, sino también aplican los conocimientos de sus disciplinas para entregar un resultado adecuado que permita descartar enfermedades o aplicar el tratamiento correcto.

\section{CRÍTICA}

Como se ha visto a lo largo de este análisis, la normativa que regula la transferencia de competencias del Indecopi a SuSalud no es lo suficientemente precisa, pues deja bastante trabajo exegético que traspasa a los funcionarios encargados de aplicarla y alcanza a los consumidores y usuarios de estos servicios quienes prácticamente se ven obligados a contratar a un abogado para decidir ante qué autoridad administrativa denunciar a fin de no perder tiempo recibiendo resoluciones o cartas de declinación de competencias.

Por otro lado, también es preciso criticar la excesiva especificidad con la que el Ministerio de Salud ha tipificado las infracciones sancionables por SuSalud contempladas en el Reglamento de Infracciones y Sanciones ${ }^{9}$, las cuales, a modo de ejemplo, van desde la no entrega de cobertura oportuna a los afiliados $o$ a sus beneficiarios ${ }^{10}$ hasta la entrega de información relativa a la atención de reclamos de sus usuarios en formato medio o cualquier otra forma distinta a la establecida, salvo que demuestre que la misma responde a fuerza mayor o caso fortuito.

De esta manera, la principal consecuencia de esta excesiva especificidad es que no es posible subsumir determinadas conductas infractoras en los supuestos establecidos en el Reglamento, pero que son sancionables bajo una interpretación amplia del Decreto Legislativo 1158. Así, casos como el de la señora Arbulú, en el que la clínica no la habría mantenido informada sobre la solicitud de cobertura por el internamiento de su menor hija no se encuentra contemplada como conducta sancionable en el Reglamento citado, pese a ser un caso evidente de falta de idoneidad en el servicio de gestión administrativa de la clínica, el cual, como ya se vio, constituye un servicio complementario que coadyuva a la prestación del servicio médico esencial de la clínica.

\section{CONCLUSIONES}

1. La aparición de SuSalud ha implicado el traslado de ciertas competencias del Indecopi en materia de servicios médicos y de seguros, lo cual no ha sido correctamente conducido, en la medida que el legislador y el Ministerio de Salud han regulado de manera deficiente las materias que son competencia de SuSalud y las que mantiene el Indecopi. Todo ello ha generado un escenario de inseguridad jurídica, tanto para los consumidores, proveedores y funcionarios públicos.

2. El Reglamento de Infracciones y Sanciones de SuSalud adolece de una falencia importantísima al carecer de una cláusula general que permita encuadrar infracciones que no pueden ser subsumidas por los demás supuestos, pero que resultan ser antijurídicas en virtud del Decreto Legislativo 1158.

3. La estructura orgánica y el Reglamento de Infracciones y Sanciones de SuSalud han predeterminado la actitud reacia de dicha entidad a atender reclamos y denuncias por violación de normas de protección al consumidor en materia de servicios médicos y productos de seguros, en la medida que ha sido diseñada principalmente para fiscalizar y supervisar a los proveedores de dichos servicios e iniciar procedimientos sancionadores de oficio por violación principalmente de su Reglamento y no por derechos de los consumidores en general (idoneidad, información y los que se señalan en los artículos 67 y siguientes del Código de Consumo).

4. Existe un espectro amplio de servicios y productos relacionados a prestaciones médicas y de seguros que deben ser analizadas caso por caso para determinar la entidad administrativa competente que conozca una eventual denuncia por falta de idoneidad o infracción a cualquier derecho o deber que establece el Código de Consumo. Proponemos como un primer esbozo para llegar a una interpretación que salve el problema en casos concretos los siguientes lineamientos:

9 Aprobado mediante Decreto Supremo N N 031-2014-SA.

10 Inciso 1 de la sección de Infracciones graves del Anexo I-A. 


\begin{tabular}{|l|l|l|}
\hline \multicolumn{1}{|c|}{ CATEGORÍA } & \multicolumn{1}{|c|}{ PRODUCTOS Y SERVICIOS ESPECÍfICOS } & \multicolumn{1}{c|}{ ENTIDAD COMPETENTE } \\
\hline \multirow{3}{*}{ Actos médicos } & $\begin{array}{l}\text { Intervenciones quirúrgicas, diagnóstico de enfermedades o trata- } \\
\text { mientos recetados }\end{array}$ & SuSalud \\
\hline \multirow{3}{*}{ Seguros y planes de salud } & SCTR y SOAT & SuSalud \\
\cline { 2 - 4 } & SCTR y SOAT solo en lo referido a prestaciones económicas & Indecopi (CC1) \\
\cline { 2 - 4 } & $\begin{array}{l}\text { Otros seguros y planes de salud, siempre que se enmarquen en al- } \\
\text { guno de los tres supuestos del artículo 9 del DL. 1158 }\end{array}$ & SuSalud \\
\hline \multirow{3}{*}{ Servicios complementarios } & $\begin{array}{l}\text { Siempre que sean previas o derivadas de la relación de consumo } \\
\text { principal entablada con una IPRESS o IAFAS }{ }^{11}\end{array}$ & SuSalud \\
\hline \multirow{3}{*}{$\begin{array}{l}\text { Otros productos para la } \\
\text { salud }\end{array}$} & $\begin{array}{l}\text { Farmacias, boticas, centros de venta de insumos químicos y equi- } \\
\text { pos médicos u odontológicos }\end{array}$ & Indecopi (CC2) \\
\cline { 2 - 4 } & Productos y servicios de medicina alternativa & Indecopi (CC2) \\
\cline { 2 - 4 } & Servicios de diagnóstico por imágenes y los laboratorios clínicos & SuSalud \\
\hline
\end{tabular}

5. Finalmente, no debemos perder de vista que el usuario o paciente es uno de los sujetos involucrados más perjudicados en este conflicto de competencia debido a las falencias en la regulación de la transferencia de funciones advertidas en el presente artículo. Las acciones a tomar para reducir el impacto negativo deben estar enfocadas en brindarle una solución eficiente y rápida, lo cual se traduce en tener normas claras y precisas y que las entidades responsables comuniquen de manera eficaz ante qué organismo público deben presentar su denuncia, ello no solo evita tramitar denuncias improcedentes, sino que, sobre todo, refleja la imagen de una administración pública organizada y que sirve a la ciudadanía.

\section{REFERENCIAS}

Bustamante Alarcón, R. (2000). El derecho a una decisión justa como elemento esencial de un proceso justo. En: Derecho \& Sociedad 15.

Brewer Carías, A. (2005). Derecho Administrativo Principios del Derecho Público, Administración Pública y Derecho Administrativo, Personalidad Jurídica en el Derecho Administrativo. Tomo I. Primera Edición. Santafé de Bogotá: Universidad Externado de Colombia.

Cassagne, J. (2010). Derecho Administrativo. Tomo I. Lima: Palestra Editores.

(1981). El acto administrativo. Segunda edición actualizada. Buenos Aires: Alfredo-Perrot.

Ezpinoza Espinoza, J. (2011). Primeras reflexiones a propósito del Código de Protección y Defensa del Consumidor. En: lus et Veritas 41.
Gordillo, A. (2000). Tratado de Derecho Administrativo. Quinta Edición. Buenos Aires: Fundación de Derecho Administrativo.

Guzmán Napuri, C. (2016). Los procedimientos administrativos sancionadores en las entidades de la Administración Pública. Lima: Gaceta Jurídica.

Marienhoff, M. (1966). Tratado de Derecho Administrativo. Volumen I. Buenos Aires: AbeledoPerrot.

Organización Mundial de la Salud. (2017). Medicina Tradicional: definiciones. Ginebra. Consulta: 6 de julio de 2017. <http://www.who.int/topics/traditional_medicine/definitions/es/>

Priori Posada, G. (2004). La competencia en el proceso civil peruano. En: Derecho \& Sociedad 22.

Rojas Montes, V. (2004). La distribución de competencias administrativas entre los organismos electorales del Perú. En: Oficina Nacional de Procesos Electorales. (ed.). La administración electoral en el Perú - Funciones, competencias y organismos electorales. Lima: Oficina Nacional de Procesos Electorales.

Sánchez-Moreno, F. (2014). El Sistema Nacional de Salud en el Perú. En: Revista Peruana de Medicina Experimental y Salud Pública 31. Consulta: 6 de julio de 2017. <http://www.scielo.org.pe/ scielo.php?pid=S172646342014000400021\&s cript=sci_arttext>

Vélez, A. (1979). Competencia y Jurisdicción Administrativa. Santafé de Bogotá: Facultad de Ciencias Jurídicas y Socioeconómicas de la Pontificia Universidad Javeriana.

11 Los servicios complementarios brindados en el marco de la atención de un producto de seguro deben enmarcarse en alguno de los tres supuestos establecidos en el artículo 9 del Decreto Legislativo 1158 
Comisión de Protección al Consumidor 1 del Indecopi. Resolución Final 1130-2017/CC1.

Resolución Final 03452017/CC1

Resolución 2280-2016/CC1

Decretos Legislativos 1033 del 25 de junio del 2008. Decreto Legislativo que aprueba la Le de Organización y funciones del Indecopi.

Decreto Legislativo 1158 del 6 de diciembre del 2013. Decreto Legislativo que dispone las medidas destinadas al fortalecimiento y cambio de denominación de la Superintendencia Nacional de Aseguramiento en Salud.

Decreto Supremo 031-2014-SA del 6 de julio del 2014. Reglamento de Infracciones y Sanciones de SuSalud.

Decreto Supremo 026-2015-SA del 13 de agosto del 2015. Reglamento de Transferencia de Funciones del Indecopi a SuSalud, en el marco del Decreto Legislativo 1158.

Decreto Supremo 030-2016-SA del 27 de julio de 2016. Reglamento para la Atención de Recla- mos y Quejas de los Usuarios de IAFAS, IPRESS y UGIPRESS.

Ley 29571. Código de Protección y Defensa del Consumidor.

Presidencia de Consejo de Ministros. Decreto Supremo 058-2011-PCM del 5 de julio del 2011.

Resolución 027-2013-INDECOPI/COD del 10 de febrero del 2013. Aprueban división temática de la competencia resolutiva de las Comisiones de Protección al Consumidor 1 y 2 de la Sede Central.

Resolución Directoral 006-2015-DIGEMID-DGMINSA del 4 de febrero del 2015. Listado de productos y servicios complementarios no autorizados en farmacias, boticas, farmacias de los establecimientos de salud y botiquines.

Resolución Final 674-2015/ILN-CPC.

Resolución 02771999/TDC-INDECOPI del 18 de agosto de 1999, seguido por Shirley Sánchez Cama contra José Cantuarias Pacheco y Corporación José R. Lindley S.A. 